http://jmscr.igmpublication.org/home/ ISSN (e)-2347-176x ISSN (p) 2455-0450

crossref DOI: https://dx.doi.org/10.18535/jmscr/v8i2.114

\title{
Study of Efficacy of Serum Lactate Dehydrogenase, Gamma Glutamyl Transpeptidase and Alkaline Phosphatase Levels as Prognostic and Diagnostic Markers in Breast Cancer
}

\author{
Authors \\ Mohammed Saheb SK ${ }^{1}$, Kasibabu $\mathrm{A}^{2}$
}

\begin{abstract}
Introduction: Carcinoma breast is one of the commonest causes of death in many developed countries in women. Cancer that is detected at an early stage can potentially be cured when the tumor is small enough to be completely removed surgically. Keeping this in view of these phenomena, the present study takes into consideration of certain biochemical investigations like Serum LDH, GGT and ALP levels that are inexpensive, accurate, and identified by the easy method of detection, and to establish their establish their diagnostic and prognostic value in breast cancer

Patients and Methods: The present study comprises of 120 participants of which 60 healthy controls, and 60 patients suffering from carcinoma breast, which was confirmed by clinical and histopathological examination.

Results: Serum LDH,GGT and ALP levels are significantly increased in breast cancer patients compared to controls and also found that the levels was gradually increased with advancing stages of breast cancer.

Conclusion: It is concluded that serum LDH, GGT and ALP levels are reliable and inexpensive promising enzyme markers with prognostic and diagnostic importance in carcinoma breast patients.
\end{abstract}

\section{Introduction}

Carcinoma breast is one of the commonest causes of death in many developed countries in women. Worldwide, there will be around 2.1 million newly diagnosed female breast cancer cases in 2018; It accounted for $24.2 \%$ of all cancers in women worldwide mortality due to breast cancer ${ }^{1}$. In India, the incidence rate of breast cancer is 25.8 per 100000 women, and mortality due to breast carcinoma is 12.7 per 100000 women $^{2}$. In India, breast cancer is one of the most common malignancies in women next to cervical cancer. A higher incidence is seen in the age group of 45-49 yrs. Multiple factors are associated with an increased risk of breast cancer, which includes genetic, environmental, and histological factors ${ }^{3}$. Genetic factors include mutation and susceptibility of genes BRCA1 and BRCA2 ${ }^{4}$; and environmental factors include early menarche, nulliparity, short lactation period, late menopause, hormone replacement therapy in which more exposure of women to circulating oestrogens ${ }^{5}$. Other risk factors include a diet high in fats, obesity, exposure to radiation, and alcohol consumption. The enzymes are the proteins present in the nucleus, cytoplasm, and mitochondria, which are released into the circulation when the cells are destroyed. Such changes in malignant tissue may result from genetic reprogramming to malignant behavior, which is likely the survival strategy of the tumors. Cancer 
that is detected at an early stage can potentially be cured when the tumor is small enough to be completely removed surgically, but a large proportion of patients of ca breast have a disease at diagnosis, which is beyond the scope of curative therapy. Keeping this in view of these phenomena and also this condition is a leading cause of death in women of developed and developing countries. The present study takes into consideration of certain biochemical investigations like Serum LDH, GGT and ALP levels that are inexpensive, accurate, and identified by the easy method of detection, and to establish their diagnostic and prognostic value in breast cancer with or without metastasis in comparison with the controls

\section{Aim}

The aim of the study is to evaluate the serum levels of LDH, GGT, and ALP to delineate the efficacy of these parameters as a diagnostic and prognostic marker in breast cancer patients.

\section{Patients and Methods}

A cross-sectional study was carried out in the Department of Biochemistry, Andhra Medical College, Visakhapatnam, with approval from the Institutional Scientific and Ethics Committee.Study was conducted between August 2018 to September 2019 with 120 subjects of age group 25 years and above were selected. All Breast cancer cases (60 Cases) are taken from General Surgery Department, and all the age matched healthy controls(60 controls) are taken from the Radiology Department, King George Hospital, Visakhapatnam, area hospital for north coastal Andhra region, Andhra Pradesh state.

\section{Inclusion Criteria}

1. All patients who were recently diagnosed as carcinoma breast clinically and histologically confirmed.

2. Patients aged from 25years and above.

3. Patients are attending the surgery OP of King George Hospital, Visakhapatnam.

4. All healthy controls are taken from the radiology department in whom mammography screening findings are negative and whose values of parameters are within the normal range.

5. Samples will be obtained from those who had given signed informed consent to participate in the study.

\section{Exclusion Criteria}

Patients suffering from, Myocardial infarction, Jaundice or liver disease, Polycythemia, Megaloblastic anemia, Hemolytic anemia, Diabetes mellitus, Renal diseases, Pancreatic diseases, Bone diseases, Severely ill patients and those who declined to give consent.

\section{Sample Collection}

Under strict aseptic precautions, $5 \mathrm{ml}$ of venous blood collected from the antecubital vein from each subject. The blood was collected in red top tubes and was allowed to clot spontaneously in a vacutiner, and then centrifuged at $3000 \mathrm{rpm}$ for 10 minutes. Whenever possible, the analysis was done immediately. When there was a delay, the samples were stored at -20-degree Celsius for further analysis. Care was taken to avoid hemolysis.

All findings were recorded, tabulated, and statistically analyzed using, unpaired t-test, and the r-test on an excel sheet and expressed in terms of mean, standard deviation, and percentage

\section{Laboratory Parameters}

- Estimation of lactate dehydrogenase (LDH) by an enzymatic kinetic method of henry. et.al (1960) ${ }^{6 .}$

- Estimation of serum gammaglutamyltranspeptidase (GGT) activity by optimized SZASZ method/IFCC method $(1986)^{7}$.

- Estimation of ALP by Kinetic photometric test, optimized standard method according to the international federation of clinical chemistry method (IFCC) ${ }^{8 .}$

\section{Results}

In the present study, the mean age of breast cancer patients was $47.3 \pm 9.17$. It was estimated that 
around $76 \%$ of the women with breast cancer are between 35-55 years of age. (Fig 1)

Fig: 1 Age-wise distribution of cases with carcinoma breast.

\section{AGE WISE DISTRIBUTION OF CASES WITH CARCINOMA BREAST}

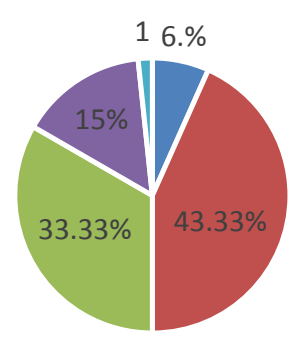

- 25-35

- 36-45

- 46-55

- 56-65

- 66-75

\section{Serum LDH levels of cases and controls}

The mean serum Lactate dehydrogenase level was $323.16 \pm 43.96 \mathrm{IU} / \mathrm{L}$ in controls, whereas in $\mathrm{Ca}$. Breast patients, it was $574.11 \pm 144.60 \mathrm{IU} / \mathrm{L}$. A significant increase in LDH was observed in ca breast patients when compared to controls with $\mathrm{p}$ value $<0.0001$. (Table 1 and Fig 2).The comparison of serum LDH levels between various stages and controls showed a significant increase between stage I and control (p-value <0.05) and a highly significant increase between stage II, stage III, stage IV, and control (p-value <0.0001). (Fig 3). An interstage comparison showed a non-significant increase in-between stage I and stage II, but a significant increase in the activity of serum LDH was observed from stage II to stage IV. (Table 2 and Fig.3)

\section{Serum GGT levels of study cases and controls}

The mean serum Gamma-glutamyl transpeptidase level was $20.67 \pm 3.83 \mathrm{IU} / \mathrm{L}$ in controls, whereas in ca breast patients, it was $57.75 \pm 13.84 \mathrm{IU} / \mathrm{L}$. A significant increase in GGT was observed in ca breast patients when compared to controls with pvalue <0.0001. (Table 1 and Fig 2). The comparison of serum GGT levels between various stages and controls showed a significant increase between stage I and control ( $p$-value <0.05) and a highly significant increase between stage II, stage III, stage IV, and control (p-value <0.0001).An interstage comparison showed a non-significant increase in-between stage I and stage II, but a significant increase in the activity of serum GGT was observed from stage II to stage IV. (Table 2 and Fig.3)

\section{Serum ALP levels of study cases and controls}

Mean serum Alkaline phosphatase level was $88.883 \pm 16.40 \mathrm{IU} / \mathrm{L}$ in controls, whereas in ca breast patients, it was $180.95 \pm 31.32$ IU/L. A significant increase in serum ALP was observed in ca breast patients when compared to controls with p-value $<0.0001$. (Table 1 and Fig 2).Serum ALP levels of all stages of ca breast patients when compared with controls, there was a highly significant increase ( $\mathrm{p}$ value <0.0001) in stage III and IV, while a significant increase in stage II ( $p$-value $<0.001$ ) and there was no significant difference in stage I ( $p$ value <0.136). (Fig.3) An interstage comparison showed a non-significant increase in-between stage I and stage II, but a significant increase in the activity of serum GGT was observed from stage II to stage IV. (Table 2 and Fig.3)

Serum GGT levels in Ca. Breast patients are positively correlated with the serum levels of $\mathrm{LDH}$ and ALP. (Table 3 and Fig 4 and 5)

Table No.1: Serum enzyme levels in controls and carcinoma breast cases and their $\mathrm{p}$-values

\begin{tabular}{|l|c|c|c|}
\hline & $\begin{array}{c}\text { Control } \\
(\text { Mean } \pm \text { SD })\end{array}$ & $\begin{array}{c}\text { Cases } \\
(\text { Mean } \pm \text { SD })\end{array}$ & p-value \\
\hline LDH(U/L) & $323.16 \pm 43.96$ & $574.75 \pm$ & $<0.0001$ \\
& & 143.74 & \\
\hline GGT(U/L) & $20.67 \pm 3.83$ & $57.75 \pm 13.84$ & $<0.0001$ \\
\hline ALP(U/L) & $88.883 \pm 16.40$ & $169.6 \pm 42.7$ & $<0.0001$ \\
\hline
\end{tabular}

Fig: 2 Comparision of Serum Levels of ALP, GGT and LDH in Controls and cases of Breast Cancer COMPARISION OF SERUM LEVELS OF ALP,GGT AND LDH IN CONTROLS AND CASES OF BREAST CANCER

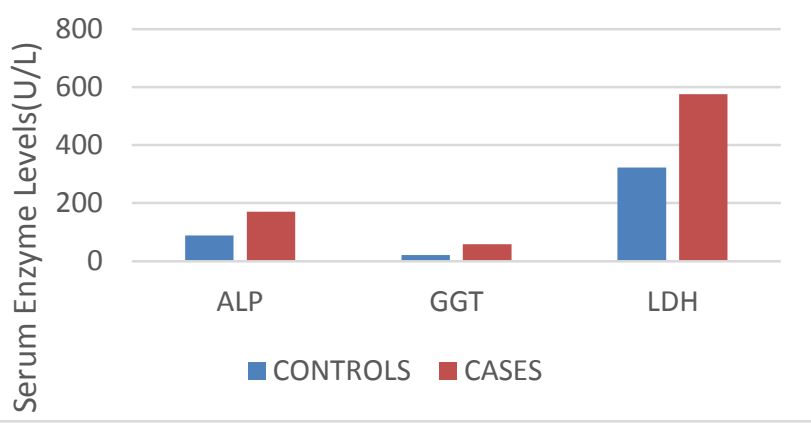




\section{JMSCR Vol||08||Issue||02||Page 661-667||February}

Fig: 3 Comparision of serum levels of ALP, GGT and LDH in different stages of breast cancer

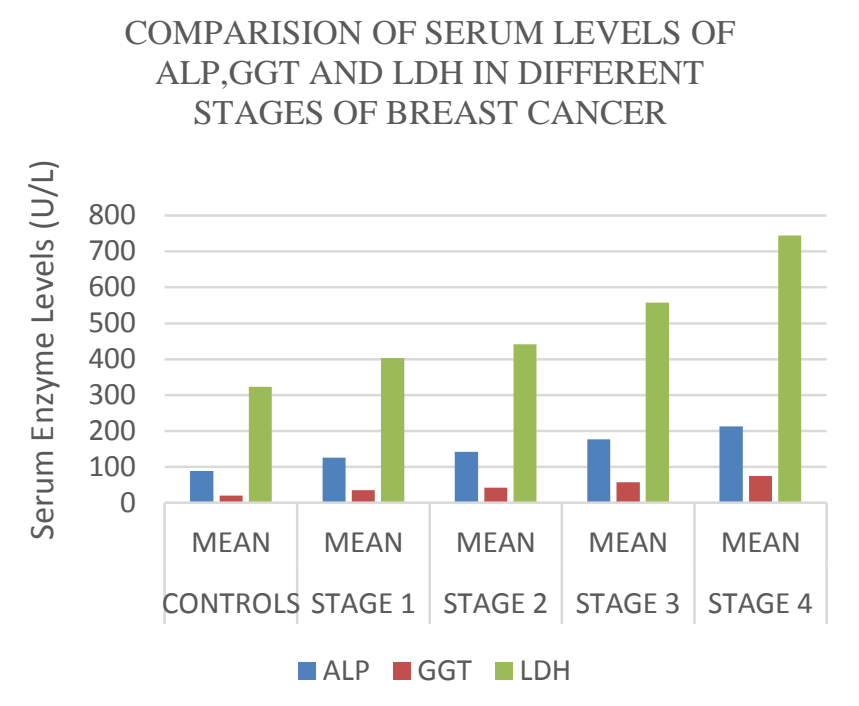

Table: 2 Comparison of LDH, GGT, and ALP in different clinical stages of breast cancer patients (ANOVA Test)

\begin{tabular}{|l|c|c|c|c|}
\hline STAGE & $\begin{array}{c}\text { NO OF } \\
\text { CASES }\end{array}$ & LDH(U/L) & GGT(U/L) & ALP(U/L) \\
\hline I & 3 & $403.33 \pm 10.06$ & $35.33 \pm 2.20$ & $107.00 \pm 4.0$ \\
\hline II & 13 & $441.76 \pm 27.18$ & $42.76 \pm 3.99$ & $115.76 \pm 6.44$ \\
\hline III & 28 & $557.89 \pm 68.56$ & $57.69 \pm 7.66$ & $176.71 \pm 20.15$ \\
\hline IV & 16 & $744.43 \pm 140.57$ & $74.23 \pm 7.08$ & $212.87 \pm 32.10$ \\
\hline ANOVA & F Value & 33.959 & 64.535 & 55.950 \\
& P- & $<0.001$ & $<0.001$ & $<0.001$ \\
& Value & & & \\
\hline & I vs. II & $>0.05(\mathrm{NS})$ & $>0.05(\mathrm{NS})$ & $>0.05(\mathrm{NS})$ \\
\hline & I vs III & $<0.001(\mathrm{HS})$ & $<0.001$ & $<0.001(\mathrm{HS})$ \\
\hline & I vs IV & $<0.001(\mathrm{HS})$ & $<0.001(\mathrm{HS})$ & $<0.001(\mathrm{HS})$ \\
\hline & II vs III & $<0.001(\mathrm{HS})$ & $<0.001(\mathrm{HS})$ & $<0.001(\mathrm{HS})$ \\
\hline & II vs IV & $<0.001(\mathrm{HS})$ & $<0.001(\mathrm{HS})$ & $<0.001(\mathrm{HS})$ \\
\hline & III vs & $<0.001(\mathrm{HS})$ & $<0.001(\mathrm{HS})$ & $<0.001(\mathrm{HS})$ \\
& IV & & & \\
\hline
\end{tabular}

NS=NON SIGNIFICANT(p-value->0.05)

HS=HIGHLY SIGNIFICANT(p-value-<0.001)

Table: 3 Correlation of GGT levels with LDH and ALP levels in Breast cancer patients. Pearson correlation coefficient (r-value)

\begin{tabular}{|l|c|c|}
\hline S.no & Parameter & r value \\
\hline 1 & LDH & +0.832 \\
\hline 2 & ALP & +0.913 \\
\hline
\end{tabular}

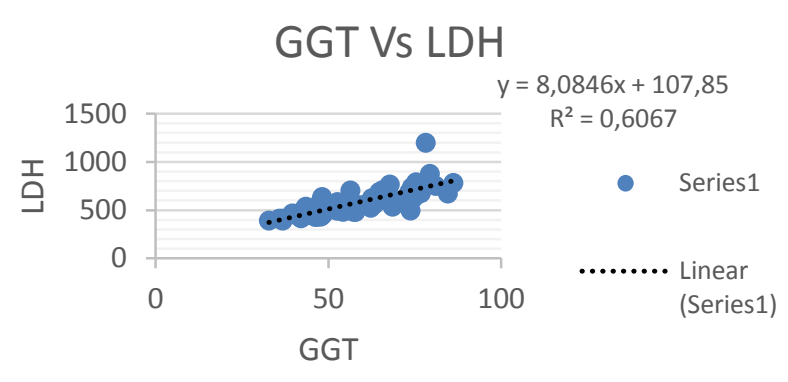

Fig: 4 Correlation of GGT levels with LDH levels in Breast cancer patients

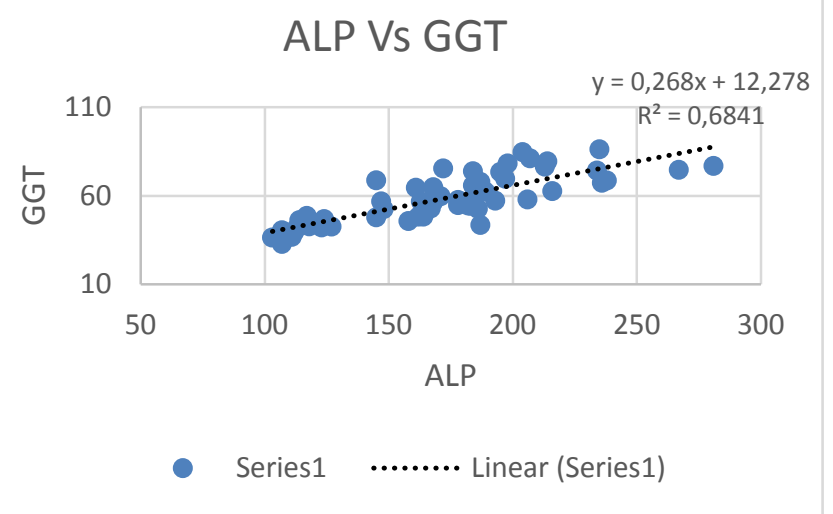

Fig: 5 Correlation of GGT levels with ALP levels in Breast cancer patients

\section{Discussion}

Breast cancer is amongst the most common malignancies in women. The view of this present study had been undertaken to assess the clinical utility of some of the promising enzyme markers Lactate dehydrogenase, Gammaglutamyltranspeptidase, and Alkaline phosphatase which are inexpensive, identified by easy methods and validated that can be of some diagnostic and prognostic significance

According to the present study, there was a significant increase in serum Lactate dehydrogenase levels with a mean value $(574.11 \pm 144.60 \mathrm{IU} / \mathrm{L})$ in $\mathrm{Ca}$ breast patients as compared to controls (323.16 \pm 43.96 IU/L). (Table 1 and Fig.2) An interstage comparison showed a non-significant increase in-between stage I and stage II, but a significant increase in the activity of serum LDH was observed from stage II to stage IV. (Table 9 and Fig.7). Present study findings are in accordance with the study conducted by Basnyat AS et al. ${ }^{9}$ Guddanti Rajeswari et al. ${ }^{10}$, in their study, 
observed serum LDH is significantly increased in $\mathrm{Ca}$ breast patients when compared to controls. In the study of Aditi Agarwal et al.$^{12}$, the serum LDH levels were found to be higher in breast cancer patients compared to controls and also corresponded with the clinical stage and bulk of the tumor which correlates with the present study. Serum LDH levels interstage Comparison shows a non-significant increase $(>0.05)$ between the $1^{\text {st }}$ and $2^{\text {nd }}$ stage but shows a significant increase in comparision with the other stages of breast cancer. (Table 9)

In present study serum LDH levels are significantly increased in breast cancer patients compared with control groups because of the high rate of anaerobic glycolysis by tumor cells, increased production of enzymes by tumor cells, change in the permeability of cells allowing leakage of soluble enzymes into circulation, and because of tumor blockade of the duct system through which the enzymes pass. The release of LDH from the destruction of tumor cells and the induction of LDH synthesis in the normal tissues of the host by the tumor also contribute to raised $\mathrm{LDH}$ levels.

In the present study, serum Gammaglutamyltranspeptidase level was significantly increased with mean value(57.75 \pm 13.84) in Ca.breast patients when compared to controls (mean=20.67 \pm 3.83 ). (Table 1 and Fig.2) When various stages of Ca.breast were compared with controls, a rise in stage I was significant ( $\mathrm{P}$ value $<0.005$ ) when compared to controls Whereas in stage II, III and IV a highly significant increase(P-value <0.0001) was observed when compared to controls. (Fig 3). Present study findings are in accordance with the study Basnyat AS et al. ${ }^{9}$, Guddanti Rajeswari et al. ${ }^{10}$, Jarari et al ${ }^{16}$ Choudhari et al. ${ }^{14}$ andChandrakanth et al. ${ }^{17----i n}$ their study observed serum GGT levels are significantly increased in $\mathrm{Ca}$ breast patients when compared to controls and also observed the further significantly increased levels of GGT (P-value <0.0001) in different stages of $\mathrm{Ca}$ breast cases compared to control subjects. In contrast, to present study Guddanti Rajeswari et al. ${ }^{10}$, in their study, there is a negative correlation ( $r$-value $=-0.2105)$ of serum
GGT with ALP levels in the post-menopausal group of breast cancer patients.

In the present study, an increase in serum GGT activity is due to the rapid turnover of malignant cells, which release the enzymes into the bloodstream. In the present study, the elevation in the serum GGT was found to be directly proportional to the advancing stage. This steady increase in the GGT activity from stage 1 to stage 4 may indicate that it can serve as a better biochemical marker.

In the present study, a significant increase in serum ALP with mean value $(176.18 \pm 35.07)$ was observed in ca breast patients when compared to controls $(88.883 \pm 16.40)$. (Table 1$)$ it was observed that there is no significant increase ( $\mathrm{P}$-value $>0.05$ ) in stage 1 but significantly increased in stage II, III, and IV (P-value<0.0001)compared to controls. Guddanti Rajeswari et.al. ${ }^{10}$, Chandrakanth $\mathrm{KH}$ et al. ${ }^{11}$, Swathi s.et al. ${ }^{13}$, and prabhasheela et al. ${ }^{15}$ have found in their study, significantly increased serum ALP levels in breast cancer patients compared to controls. (Table 1) When all four stages were individually compared with control, we found that the alkaline phosphatase level was gradually increased with advancing stages. This is in agreement with the present study that serum ALP levels were found to be significantly increased with advancing stages of breast cancer.

Elevation of serum ALP in Ca. Breast occurs due to neoplastic infiltration of the liver resulting from localized intrahepatic cholestasis with increased enzyme synthesis in liver tissue adjacent to the neoplasm, though pressure necrosis of liver cells and enzyme formation in proliferating endothelial cells may also be contributory.

\section{Limitations of the study}

The present study was conducted on a small group of women with carcinoma breast. Further studies on a larger sample with longer follow up are needed to substantiate our findings before conclusions can be drawn on the utility of these parameters for the diagnosis and prognosis in carcinoma breast patients. 


\section{Conclusion}

In conclusion, the present study suggests that serum Lactate dehydrogenase,

Gammaglutamyltranspeptidase and Alkaline phosphatase could be better biomarkers for diagnosing carcinoma breast and for monitoring its progression and treatment. Serial analysis using plasma Alkaline phosphatase isoenzymes combined with other parameters like Gamma-glutamyltranspeptidase (GGT) for the detection of metastasis would seem to be justified. As per the present study, there is increased incidence of breast cancer has been observed in post-menopausal patients and accounts for high morbidity and mortality. The serial measurement of these enzymes will have a prognostic significance and help treatment decisions. Such measurements, though less sensitive than imaging procedures, together with clinical and histopathological findings, may serve as potential biomarkers that are accurate, sensitive, cost-effective, and can be easily assayed in smaller laboratories that have not yet exposed to any sophisticated technology.

\section{References}

1. Freddie Bray; Jacques Ferlay; Isabelle Soerjomataram; Rebecca L. Siegel.et.al; Global Cancer Statistics 2018: GLOBOCAN Estimates of Incidence and Mortality Worldwide for 36 Cancers in 185 Countries; CA CANCER J CLIN 2018;68:394-424.

2. Gupta A, Shridhar K, Dhillon PK. A review of breastcancer awareness among women in india: cancer literate or awareness deficit Eur J Cancer 2015; 51: 2058-66.

3. Collaborative Group on Hormonal Factors in Breast Cancer: Menarche, menopause, and breast cancer risk: individual participant meta-analysis, including 118964 women with breast cancer from 117 epidemiological studies. Lancet Oncol 2012; 13:1141-51.

4. Ford D, Easton DF, Stratton M. et. al. Genetic heterogeneity and penetrance analysis of the BRCA1 and BRCA2 genes in breast cancer families. Am J Hum Genet. 1998; 62:676-89.

5. Brinton LA, Richesson D, Leitzmann MF, Gierach GL, Schatzkin A, Mouw T, et al. Menopausal hormone therapy and breast cancer risk in the NIH-AARP. Diet and Health Study Cohort. Cancer Epidemiol Biomarkers Prev. 2008; 17:3150-60.

6. Henry RJ, Chiamor N, Golub OJ, Berkman S. Revised spectrophotometric methods for the determination of glutamic -oxaloacetic transaminase and lactic acid dehydrogenase. Am J ClinPathol. 1960; 34: 381-98.

7. Szasz, G., Clinical chemistry, Vol.22, No.12 (1976), 2051.

8. Bowers, G.N., and McComb, R.B., Clin Chem. 21: 1988 -1995, 1975.

9. Basnyat AS, Bhupal Govinda Sreshta, Abhimanyu Jha, R Pathak Study of Serum Lactate Dehydrogenase and GammaGlutamyl Transpeptidase in Breast Cancer Patients Receiving Chemotherapy .The journal of tropical science, April 2017 Vol. 7, No. 2, pp. $128-132$.

10. Guddanti Rajeswari, Psatyasrinivas, k. sivarama Krishna sai, Edalasuresh/ International Journal of Biomedical and Advance Research 2016; 7(1): 031-034.

11. Chandrakanth KH. K pyati, A,murthy DS, J. Significance of serum total alkaline phosphatase levels in breast cancer. Int $\mathbf{J}$ Clin and Biomed Res. 2016;2(1):13-15.

12. Aditi Agrawal et al., LDH Biomarker in Ca. Breast. Journal of Clinical and Diagnostic Research. 2016 Mar, Vol-10(3): BC06BC08.

13. Swati S, Neelima Singh, Akshay Kumar N, Kumar S, Sanjay Singh, Reetika S. Serum Alkaline Phosphatase Level As A Better Predictor for Metastatic Breast Cancer in Comparison to Acid Phosphatase And Calcium Activities, Journal of Dental and Medical Sciences (IOSR-JDMS). (December. 2016), Volume 15, Issue 12 Ver. II PP 15-19. 
14. Choudhari A, Desai P, Indumati V, Kadi S. Activities of serum Ada, GGT and alp in carcinoma breast-a case control study for diagnostic and prognostic significance .Indian J Med Sci 2013;67:123-9.

15. Prabasheela B, S Baskaran, R Arivazhagan. Evaluation of alkaline phosphatase in pre and post operative breast cancer patients. Int J Biol Med Res. 2012; 3(2): 1536-1537.

16. M Jarari Abdalla, S.shakila, Saeid Omar Alsoaeitiv, Nouh. MH. Aljarari et.al. Serum levels of LDH and Gamma GT in Libyan Breast Cancer patients. Indian journal of Medical Research. Volume: 3 | Issue : 12 | Dec 2013.

17. Chandrakanth KH, Nagaraj, Murthy JDS, Kumar SD, Pyati A. Study of serum levels of gamma- glutamyltransferase, lactate dehydrogenase malondialdehyde and vitamin $\mathrm{E}$ in breast cancer. Int $\mathrm{J}$ of Pharma and Bio Sciences. 2(4):489-98, OctDec 2011. 